\title{
Etude de dermatophytes isolés dans les services de revalidation et physiothérapie d'une structure hospitalière en région Liégeoise (Belgique)
}

R. Sacheli ${ }^{1}$, T. Utri² ${ }^{2}$ A. Hidjababdoulaye ${ }^{2}$, J. Alfageme-Gonzalez, C. Adjetey ${ }^{2}$, M-P. Hayette ${ }^{1}$

${ }^{1}$ Centre National de Référence pour les Mycoses, Département de Microbiologie Clinique, Centre Hospitalier Universitaire de Liège ${ }^{2}$ Département de Microbiologie Clinique, Centre Hospitalier Universitaire de Liège ${ }^{3}$ Département d'Hygiène Hospitalière, Centre Hospitalier Universitaire de Liège.

\section{Objectifs}

La présence de dermatophytes en milieu sportif (salles de sport, piscine, tatamis), douches collectives, vestiaires des écoles, a été largement décrite. Par contre il existe peu de littérature concernant les établissements de soins. C'est pourquoi, nous avons fait une étude de surveillance en milieu hospitalier et particulièrement dans les salles de kinésithérapie et de revalidation ainsi que dans une piscine d'établissements du CHU de Liège.

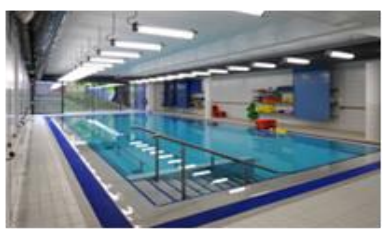

Collecte des souches sur trois sites sportifs hospitaliers le site du Sart Tilman (ST), le site Ourthe Amblève (OA) et le site Notre-Dame des Bruyères (NDB)

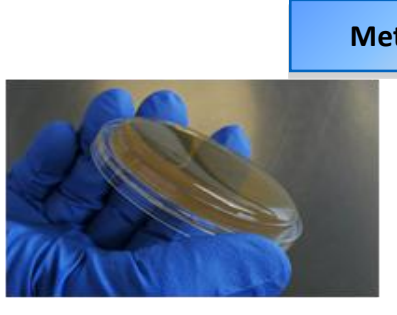

Prélèvements sur gélose de contact Sabouraud, Chloramphénicol. gentamycine, actidione (Tritium Microbiologie)
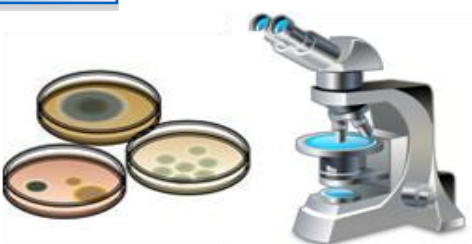

Identification macro/ microscopique

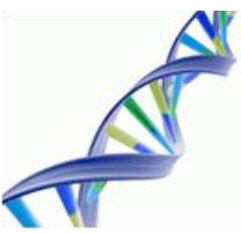

Sequençage ITS pour confirmation

\section{Resultats}

$>$ Au total, 552 prélèvements ont été réalisés parmi lesquels 15 dermatophytes ont été recensés (2,72\%). On retrouve: 7 T. rubrum, 7 T. interdigitale, 1 T. mentagrophytes var. mentagrophytes (Fig.1).

$>$ La répartition des espèces est rassemblée dans le tableau 1.

$>$ D'autres champignons non dermatophytes potentiellement pathogènes ont été isolés des trois sites hospitaliers dont, Alternaria sp., Chrysosporium sp., Fusarium sp. et Scopulariopsis sp. Onychocola canadensis a été isolé à partir de de l'eau de piscine (Site OA) (Fig.2).

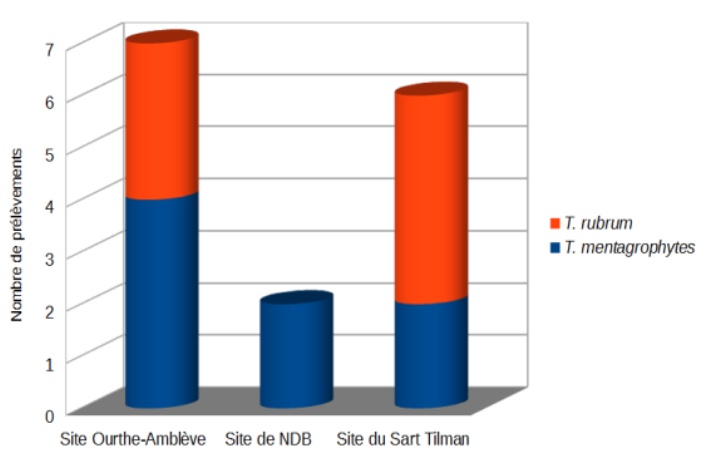

Figure 1:Distribution des espèces de dermatophytes retrouvés sur les sites ST, OA et NDB.

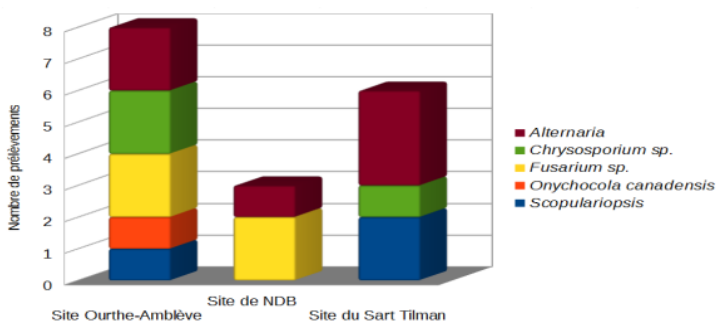

Figure 2: Distribution des champignons non dermatophytes potentiellement pathogènes isolées des sites ST, OA et NDB.

\begin{tabular}{|c|c|c|c|}
\hline \multicolumn{4}{|c|}{ Esneux $(O A), 226$ prélèvements } \\
\hline Espèce isolée & Lieux de prélèvement & $\begin{array}{l}\text { Nombre de } \\
\text { colonies par } \\
\text { boîte }\end{array}$ & \begin{tabular}{|l} 
Nombre de \\
colonies par $\mathrm{m}^{2}$
\end{tabular} \\
\hline $\begin{array}{l}\text { Trichophyton } \\
\text { mentagrophytes }\end{array}$ & Sol d'un vestiaire à la piscine avant nettoyage & 2 & 783,7 \\
\hline \begin{tabular}{|l} 
Trichophyton \\
mentagrophytes
\end{tabular} & Pédale gauche d'un vélo & 1 & 391,8 \\
\hline $\begin{array}{l}\text { Trichophyton } \\
\text { mentagrophytes }\end{array}$ & Pédale droite d'un rameur & 4 & 1567,4 \\
\hline $\begin{array}{l}\text { Trichophyton } \\
\text { mentagrophytes }\end{array}$ & Douche dans le vestiaire commun dames (sol) & 1 & 391,8 \\
\hline Trichophyton rubrum & Sol d'un vestiaire à la piscine avant nettoyage & 1 & 391,8 \\
\hline Trichophyton rubrum & Balance & 1 & 391,8 \\
\hline Trichophyton rubrum & Table de kinésithérapie dans un box de consultation & 1 & 391,8 \\
\hline \multicolumn{4}{|c|}{ Notre Dame des Bruyères (NDB) 119 prélèvements } \\
\hline \begin{tabular}{|l} 
Trichophyton \\
mentagrophytes
\end{tabular} & Tapis de gymnastique & 2 & 783,7 \\
\hline $\begin{array}{l}\text { Trichophyton } \\
\text { mentagrophytes }\end{array}$ & Pédale droite d'un vélo & 1 & 391,8 \\
\hline \multicolumn{4}{|c|}{ Sart Tilman (ST) 207 prélèvements } \\
\hline $\begin{array}{l}\text { Trichophyton } \\
\text { mentagrophytes }\end{array}$ & Filet d'un trampoline & 1 & 391,8 \\
\hline $\begin{array}{l}\text { Trichophyton } \\
\text { mentagrophytes }\end{array}$ & Tapis de gymnastique & 1 & 391,8 \\
\hline Trichophyton rubrum & Douche dans les vestiaires hommes (sol) & 4 & 1567,4 \\
\hline Trichophyton rubrum & Demi-sphère d'équilibre & 1 & 391,8 \\
\hline Trichophyton rubrum & Sol des vestiaires hommes & 6 & 2351,1 \\
\hline Trichophyton rubrum & Tapis de gymnastique & 1 & 391,8 \\
\hline
\end{tabular}

Table 1: Tableau décrivant les lieux de prélèvements positifs pour des espèces dermatophytes sur les trois sites hospitaliers ST, OA et NDB. Le nombre de colonies retrouvé sur les boites de contact ainsi qu'une extrapolation du nombre de colonies par $\mathrm{m} 2$ sont également décrits.

\section{Conclusions}

Grâce à cette étude, nous avons pu mettre en évidence sur le matériel de revalidation et les sols des vestiaires, la présence de deux espèces anthropophiles, $T$. rubrum et $T$. interdigitale fréquemment impliquées dans les cas d'onychomycoses et atteintes cutanées. Une souche zoophile de $T$. mentagrophytes a également été mise en évidence. Le taux de contamination par les dermatophytes s'élève à 2,72\%. Cette étude démontre qu'un renforcement de la fréquence de nettoyage des sols et des instruments utilisés dans les salles de sport du CHU de Liège est nécessaire de façon à minimaliser les risques de contamination de patients et du personnel médical. 